\title{
Dietary Behaviors in the Post-Lockdown Period and Its Effects on Dietary Diversity: The Second Stage of a Nutrition Survey in a Longitudinal Chinese Study in the COVID-19 Era
}

\author{
Jian Zhang ${ }^{1,2}$, Ai Zhao ${ }^{1, *}$, Yalei Ke ${ }^{2}$, Shanshan Huo ${ }^{2}$, Yidi Ma ${ }^{2}$, Yumei Zhang ${ }^{2}$, Zhongxia Ren ${ }^{2}$, \\ Zhongyu $\mathrm{Li}^{3}$ and Keyang Liu ${ }^{4}$ \\ 1 Vanke School of Public Health, Tsinghua University, Beijing 100091, China; Jianzhang_bjmu@163.com \\ 2 School of Public Health, Peking University, Beijing 100191, China; yaleike_bjmu@163.com (Y.K.); \\ shanshanhuo_bjmu@163.com (S.H.); yidima_bjmu@163.com (Y.M.); yumeizhang_bjmu@163.com (Y.Z.); \\ zhongxiaren_bjmu@163.com (Z.R.) \\ 3 Department of International Health, Johns Hopkins Bloomberg School of Public Health, \\ Baltimore, MD 21205, USA; zli132@jhmi.edu \\ 4 Public Health, Department of Social Medicine, Osaka University Graduate School of Medicine, \\ Osaka 5620032, Japan; liu@pbhel.med.osaka-u.ac.jp \\ * Correspondence: aizhao18@tsinghua.edu.cn; Tel.: +86-138-1113-1994
}

Received: 30 September 2020; Accepted: 22 October 2020; Published: 26 October 2020

Abstract: Coronavirus disease 2019 (COVID-19) has imposed enormous challenges on people's lifestyles. People in China have gradually returned to normal life; however, in the protracted pandemic, people may still follow certain dietary behaviors to cope with COVID-19. This study was the second stage of a longitudinal nutritional survey conducted in post-lockdown China that was aimed at exploring post-lockdown dietary behaviors and their effects on dietary diversity. In line with the first stage of the survey, the current dietary behaviors used to cope with COVID-19 and ways of purchasing food were determined. In addition, changes in dietary behavior compared to the same period in 2019 and those behaviors recommended to ensure food safety were also investigated. The Household Dietary Diversity Score (HDDS) was used to assess dietary diversity; this was also used in the first stage of the survey. Linear regression was used to model the associations between the HDDS, participants' characteristics, and dietary behaviors. The data of 1994 participants were included in the analysis. The overall mean HDDS was $9.2 \pm 2.0$. Compared to the same period in 2019, a substantial proportion of participants self-reported that they had recently decreased eating in restaurants $(61.6 \%)$ and reduced intakes of seafood (53.1\%), imported frozen food (57.1\%), and raw food $(60.5 \%)$, while $64.8 \%$ of participants reported increased cooking at home. People with an increased consumption of seafood (adjusted OR $(95 \% \mathrm{CI})=0.56(0.07,1.04))$ and raw food (adjusted OR $(95 \% \mathrm{CI})=0.74(0.27,1.21))$ had a significantly higher HDDS. Participants who changed their consumption of imported frozen food (both increased and decreased) had a higher HDDS (adjusted OR $(95 \% \mathrm{CI})=0.56(0.07,1.04)$ and $0.27(0.09,0.44)$, respectively). People who depended more on purchasing food online had a significantly higher HDDS (adjusted OR $(95 \% \mathrm{CI})=0.29(0.02,0.55)$ ). Compared to the data from stage 1 , the proportion of people choosing healthy products to cope with COVID-19 did not greatly change and those people had a higher HDDS (adjusted OR $(95 \% \mathrm{CI})=0.31$ $(0.19,0.42))$. Although this study found that the proportion of people who chose to use alcohol or vinegar to prevent COVID-19 had decreased substantially compared to during lockdown, there were still 5.3\% and 9.8\% who followed these irrational behaviors. Regarding the dietary behavior regarding food safety, except for cooking food fully, fewer than half of participants followed the recommended dietary behaviors, including individual food servings (44.2\%), using serving chopsticks and spoons $(44.8 \%)$, and preparing raw and cooked food separately (43.3\%). People who followed these behaviors had a better dietary diversity. In conclusion, during the post-lockdown period, people still followed 
certain dietary behaviors to cope with COVID-19. While some dietary behaviors were adopted to help prevent infection, irrational dietary behaviors were still followed. These behaviors were associated with the dietary diversity in Chinese adults.

Keywords: COVID-19; dietary diversity; post-lockdown; dietary behaviors; China

\section{Introduction}

Declared as a pandemic by the World Health Organization (WHO), coronavirus disease 2019 (COVID-19) imposes enormous challenges on health systems, the economy, and food supplies globally, and has a great influence on individuals' lifestyles [1,2]. Chinese residents living both in and outside Hubei had been required to stay at home to self-isolate since 23 January 2020 [3]. After 76 days, on April 8, Wuhan reopened transportation connections to areas outside the city, which marked the end of the lockdown in most parts of China [3]. However, relapses of the domestic COVID-19 epidemic occasionally occurred in some places, such as Beijing, Xinjiang, and Ruili (Yunnan province) [4-6]. In the protracted COVID-19 epidemic, certain behaviors were still recommended to residents to prevent infection and maintain health, such as keeping social distancing, wearing masks, and following certain dietary behaviors [7].

In the lockdown time, we conducted the first stage of a nutrition survey on Chinese residents. Based on the data from the first stage of the survey and studies performed outside China during the pandemic, we found people's dietary behaviors to be greatly affected by COVID-19. Some people chose to eat more snacks and other high-calorie foods to cope with the depression and anxiety triggered by the pandemic, and some intended to prevent coronavirus through drinking alcohol and vinegar [8-12]. The ways to purchase food were also changed by the restriction of outside activities and transportation [8]. These changes in dietary behaviors more or less affected people's health. Although according to the data from the first stage of the survey, the dietary diversity was generally good [8], the inappropriate dietary behaviors may still raise several nutritional concerns. One previous study reported that the increased intake of high-calorie food contributed to a significant gestational weight gain in women [13]. Iran reported there were at least 2197 people poisoned and 244 deaths due to drinking toxic alcohol (methanol-based beverages) that was believed to prevent COVID-19 [14].

Currently, people in China have gradually returned to normal work and daily life. It is interesting to know whether the dietary behaviors of Chinese people are still affected by COVID-19 and its potential effects on health. In addition, to prevent COVID-19 infection, certain dietary behaviors were encouraged for the Chinese people that are significantly different from those expressed in Chinese culture, such as using serving chopsticks and spoons and serving food individually [15]. Meanwhile, because of the newly diagnosed COVID-19 cases in Beijing being linked to the market and the frozen food sold in the market [16], some professionals suggested that there is a potential risk of infection associated with exposure to frozen food [17]. How these recommendations and suggestions could influence people's dietary behaviors and further affect the nutrition status in post-lockdown times needed to be explored.

In order to observe the changes in dietary behavior of Chinese residents during the COVID-19 pandemic and to explore the potential dietary behavior that could affect health, the second stage of a longitudinal online nutrition study was conducted in August in China. Data on dietary behaviors and dietary diversity were both investigated and compared with the results of the first stage of the survey conducted during the lockdown. 


\section{Materials and Methods}

\subsection{Study Design and Participants}

This paper describes the second stage of a longitudinal nutrition study during the COVID-19 pandemic in China. The first stage was conducted in March 2020 during the lockdown. The details of the methodologies used in the first stage of the survey were described previously [8]. The second stage was conducted in August 2020 (during the post-lockdown period) through a Wenjuanxing e-questionnaire platform (Wenjuanxing Tech Co. Ltd, Changsha, China), which is widely used in China. A multistage sampling method was applied. We purposely selected persons living in northern, southern, and median parts of China. More participants were then reached using a "snowball sampling" method.

The e-questionnaire was logistically set to ensure all the participants were aged from 18 to 80 years, and there were no missing data on key questions. A total of 2267 Chinese residents responded to the survey. After examining all the collected data, those living outside mainland China $(N=9)$ were excluded. In addition, two questions were used in the questionnaire to help identify whether participants had seriously answered the questions or not. One question was placed at the end of the survey, which was: "Have you answered the questions seriously?". Another was hidden in the middle of the questionnaire: "Please choose the option "very" for this question." Those who chose "no" in the first question and did not answer "very" to the second question were considered as not responding seriously to the survey and were excluded from the data analysis $(N=264)$. Participants could get a monetary reward after the quality of response was checked by investigators.

Finally, the data of 1994 participants in the second stage of the survey were used in this study. A total of $24.2 \%$ of the participants that took part in the second stage of the survey also took part in the first stage [8]. The geographic distribution of the participants in stage two can be seen in Figure 1.

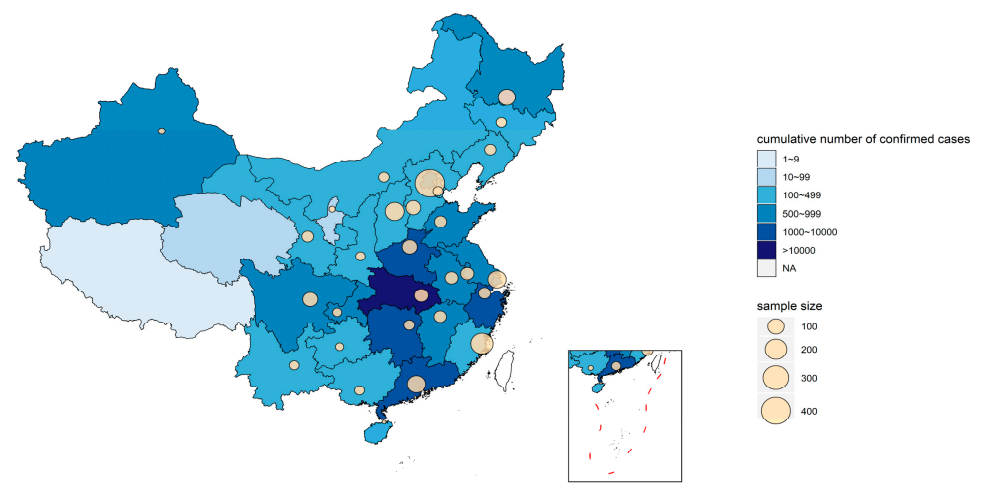

Figure 1. Geographical distribution of the studied population in the second nutrition survey wave. Figure legends: The color of the map indicates the cumulative number of confirmed cases in each area by the end of August 31, according to the report from the Chinese Health Commission [16]. A bubble plot was then generated, with the bubble size representing the number of participants at each investigation point.

\subsection{Data Collection}

There were four parts to the questionnaire: socio-demographic characteristics, COVID-19 prevalence status in different areas, dietary diversity, and dietary behaviors.

The data of the COVID-19 prevalence status, including the numbers of confirmed cases (the imported cases were not excluded) in each province, autonomous region, or municipality directly under the central government by the end of August and whether the participants' living areas had a second outbreak, were obtained from the website of the Chinese Health Commission [18].

The dietary diversity was evaluated using the Household Dietary Diversity Score (HDDS), which was developed to measure a household's access to food [8]. The consumption of 12 food 
items over the previous $24 \mathrm{~h}$ was investigated, including cereals; roots and tubers; vegetables; fruit; meat, poultry, and offal; eggs; fish and seafood; pulses, legumes, and nuts; dairy products; oils and fats; sugar and honey; miscellaneous (such as condiments, snacks, and beverages). Participants were assigned "one" or "zero" points for each food item depending on whether or not they had consumed it in the previous $24 \mathrm{~h}$. The HDDS total ranges from 0 to 12 .

A series of questions regarding changes in dietary behaviors were included. The studied population was required to self-report whether they showed increased, unchanged, or decreased frequencies of certain dietary behaviors compared to the same period in 2019, such as eating outside, cooking at home, consumption of seafood, and consumption of imported frozen food. In line with the first stage of the survey, participants were asked whether they currently had specific dietary behaviors to cope with COVID-19, including intentionally taking dietary supplements (vitamin C, probiotics, and other dietary supplements), functional food (such as ginseng and Ganoderma), and food (including vinegar and alcohol). Whether the participants followed the updated recommended behaviors after the COVID-19 outbreak were also investigated, including individual food servings, using serving chopsticks and spoons, fully cooking food, and preparing raw and cooked food separately.

To investigate food purchasing sources, consistent with questions used in the first stage of the survey, four common approaches to obtaining or purchasing different types of foods (the 12 food items in the HDDS) were asked about, namely, (1) in-house storage, (2) in-person grocery shopping, (3) using online food ordering and delivery services (including purchasing both raw ingredients and prepared meals from restaurants), and (4) being dependent on government- or community-based food distribution.

\subsection{Ethics}

The questionnaire was filled in anonymously. Informed consent was required prior to the survey by clicking the "agree" option to confirm the willingness to participate voluntarily in the survey.

\subsection{Statistics}

Data were analyzed using SAS v9.4 (SAS Institute, Cary, NC, USA). Data were presented as mean \pm standard deviation (SD) or a percentage. The comparisons of the HDDSs for participants with different characteristics were tested with an independent $t$-test or ANOVA analysis.

A linear regression model was used to explore the associations of participants' dietary behaviors with the HDDS after adjusting for the family income and living in an area with a second outbreak of COVID-19. Regarding the food purchasing pattern, the clustering process was conducted with the flexclust package within R 4.0.2. (R Core Team, Vienna, Austria) We first ran a K-means cluster analysis on the data collected in the first stage and identified three patterns of food-collecting propensity. Subsequently, we ran the "predict()" function to cluster the data collected in the second stage using the centroids from the previous analysis. Finally, three food purchasing patterns were explored and the differences in the HDDSs between the three different patterns were examined. A $p$-value $<0.05$ was considered statistically significant in all analyses.

\subsection{Heat Map}

The heat map of the geographical distribution of participants were created using R v3.6.3 (R Core Team, Vienna, Austria) with the packages "ggplot2," "maptools," "rgdal," and "sp." The base map was obtained as a shape file from the National Fundamental Geographic Information System, China. The cumulative number of confirmed cases of COVID-19 were obtained at the province, autonomous region, or municipality directly under the central government level and identified with different colors, where the darker the color, the more cases. A bubble plot was then generated, in which the bubble size represented the number of studied samples. 


\section{Results}

\subsection{The HDDSs of Participants with Different Characteristics}

A total of 1994 adults participated in this study, where $483(24.2 \%)$ had also been enrolled in stage 1 of the nutrition survey. Comparing the socio-demographic characteristics of participants in stages 1 and 2 showed the participants in stage 2 were relatively young, living in urban areas, and with both higher rates of lower (senior high school or under) and higher (master's degree or above) education levels (Table S1).

The average HDDS was 9.2 \pm 2.0. During the post-lockdown period, people with a higher family income showed a significant higher HDDS (Table 1). Other socio-demographic characteristics were not associated with the HDDS. The calculated number of confirmed cases of COVID-19 ( $\geq 500$ or $<500)$ and the occurrence of a second outbreak of COVID-19 were not associated with the HDDS.

Table 1. Household Dietary Diversity Scores (HDDSs) of participants with different characteristics.

\begin{tabular}{|c|c|c|c|c|}
\hline \multicolumn{2}{|c|}{ Characteristics of Participants } & \multirow{2}{*}{$\begin{array}{c}N \\
1778 \\
216\end{array}$} & \multirow{2}{*}{$\begin{array}{c}\text { HDDS (Mean } \pm \text { SD) } \\
9.2 \pm 2.0 \\
9.4 \pm 1.9\end{array}$} & \multirow{2}{*}{$\begin{array}{c}p \\
0.533\end{array}$} \\
\hline Age & $\begin{array}{l}18-45 \text { years old } \\
>45 \text { years old }\end{array}$ & & & \\
\hline Gender & $\begin{array}{c}\text { Male } \\
\text { Female }\end{array}$ & $\begin{array}{c}742 \\
1252\end{array}$ & $\begin{array}{l}9.2 \pm 1.9 \\
9.2 \pm 2.0\end{array}$ & 0.743 \\
\hline Education level & $\begin{array}{l}\text { Senior high school or under } \\
\text { Bachelor's degree } \\
\text { Master's degree or above }\end{array}$ & $\begin{array}{c}389 \\
1151 \\
454\end{array}$ & $\begin{array}{l}9.2 \pm 2.1 \\
9.2 \pm 1.9 \\
9.1 \pm 1.9\end{array}$ & 0.528 \\
\hline Family annual income (Chinese yuan) & $\begin{array}{c}<30 \text { thousand } \\
30-100 \text { thousand } \\
>100-300 \text { thousand } \\
>300 \text { thousand }\end{array}$ & $\begin{array}{l}215 \\
695 \\
765 \\
319\end{array}$ & $\begin{array}{l}8.6 \pm 2.2 \\
9.1 \pm 2.0 \\
9.3 \pm 1.9 \\
9.6 \pm 1.8\end{array}$ & $<0.001$ \\
\hline Geographic region & $\begin{array}{l}\text { Urban } \\
\text { Rural }\end{array}$ & $\begin{array}{c}1645 \\
349\end{array}$ & $\begin{array}{l}9.3 \pm 1.9 \\
8.8 \pm 2.1\end{array}$ & 0.067 \\
\hline Vulnerable person living in the house ${ }^{a}$ & $\begin{array}{l}\text { Yes } \\
\text { No }\end{array}$ & $\begin{array}{c}13 \\
1981\end{array}$ & $\begin{array}{c}10.3 \pm 1.9 \\
9.2 \pm 2.0\end{array}$ & 0.752 \\
\hline Confirmed cases in the province & $\begin{array}{l}<500 \\
\geq 500\end{array}$ & $\begin{array}{c}682 \\
1312\end{array}$ & $\begin{array}{l}9.2 \pm 2.0 \\
9.2 \pm 1.9\end{array}$ & 0.453 \\
\hline Second COVID-19 outbreak & $\begin{array}{l}\text { Yes } \\
\text { No }\end{array}$ & $\begin{array}{c}669 \\
1325\end{array}$ & $\begin{array}{l}9.2 \pm 2.0 \\
9.2 \pm 1.9\end{array}$ & 0.932 \\
\hline
\end{tabular}

a The vulnerable people were children under 5 years old, elders above 65 years old, and pregnant and lactating women.

\subsection{Dietary Behaviors and Their Association with the HDDS}

A substantial proportion of participants self-reported that they recently decreased their eating at restaurants $(61.6 \%)$ and reduced their intake of seafood (53.1\%), imported frozen food (57.1\%), and raw food $(60.5 \%)$, while $64.8 \%$ of participants reported increased their amount of cooking at home in comparison to the same period in the previous year (2019).

In the univariate analysis, the people who increased their consumption of seafood and raw food had a significantly higher HDDS. Participants who changed their consumption of imported frozen food (both increased and decreased) had a higher HDDS (Table 2). 
Table 2. Comparison of the HDDSs of participants with different behaviors.

\begin{tabular}{|c|c|c|c|c|}
\hline \multicolumn{2}{|c|}{ Behaviors Comparing with 2019} & \multirow{2}{*}{$\begin{array}{c}N \\
247\end{array}$} & \multirow{2}{*}{$\begin{array}{c}\text { HDDS (Mean } \pm \text { SD) } \\
9.2 \pm 2.1\end{array}$} & \multirow[t]{2}{*}{$p$} \\
\hline \multirow{3}{*}{ Eating at restaurants } & Increased & & & \\
\hline & Stay the same & 519 & $9.2 \pm 2.0$ & \multirow[t]{2}{*}{0.907} \\
\hline & Decreased & 1228 & $9.2 \pm 1.9$ & \\
\hline \multirow{3}{*}{ Eating takeaways } & Increased & 420 & $9.3 \pm 2.1$ & \multirow{3}{*}{0.469} \\
\hline & Stay the same & 583 & $9.1 \pm 2.0$ & \\
\hline & Decreased & 991 & $9.2 \pm 1.9$ & \\
\hline \multirow{3}{*}{ Cooking at home } & Increased & 1292 & $9.3 \pm 1.9$ & \multirow{3}{*}{0.286} \\
\hline & Stay the same & 514 & $9.1 \pm 2.0$ & \\
\hline & Decreased & 188 & $9.1 \pm 2.2$ & \\
\hline \multirow{3}{*}{ Purchasing food from a market } & Increased & 599 & $9.3 \pm 1.9$ & \multirow{3}{*}{0.623} \\
\hline & Stay the same & 727 & $9.2 \pm 1.9$ & \\
\hline & Decreased & 668 & $9.2 \pm 2.0$ & \\
\hline \multirow{3}{*}{ Purchasing food online } & Increased & 824 & $9.4 \pm 1.9$ & \multirow{3}{*}{$<0.001$} \\
\hline & Stay the same & 633 & $9.1 \pm 2.0$ & \\
\hline & Decreased & 537 & $9.0 \pm 2.1$ & \\
\hline \multirow{3}{*}{ Consuming seafood } & Increased & 189 & $9.6 \pm 1.9$ & \multirow{3}{*}{0.004} \\
\hline & Stay the same & 747 & $9.2 \pm 1.9$ & \\
\hline & Decreased & 1058 & $9.1 \pm 2.0$ & \\
\hline \multirow{3}{*}{ Consuming frozen food } & Increased & 377 & $9.3 \pm 1.9$ & \multirow{3}{*}{0.396} \\
\hline & Stay the same & 799 & $9.1 \pm 2.0$ & \\
\hline & Decreased & 818 & $9.2 \pm 2.0$ & \\
\hline \multirow{3}{*}{ Consuming imported frozen food } & Increased & 67 & $9.7 \pm 2.1$ & \multirow{3}{*}{0.002} \\
\hline & Stay the same & 788 & $9.0 \pm 1.9$ & \\
\hline & Decreased & 1139 & $9.3 \pm 2.0$ & \\
\hline \multirow{3}{*}{ Consuming raw food } & Increased & 69 & $9.9 \pm 2.2$ & \multirow{3}{*}{0.005} \\
\hline & Stay the same & 718 & $9.1 \pm 1.9$ & \\
\hline & Decreased & 1207 & $9.2 \pm 2.0$ & \\
\hline \multirow{3}{*}{ Consuming snacks and beverages } & Increased & 497 & $9.2 \pm 2.0$ & \multirow{3}{*}{0.587} \\
\hline & Stay the same & 749 & $9.2 \pm 2.0$ & \\
\hline & Decreased & 748 & $9.1 \pm 2.0$ & \\
\hline
\end{tabular}

Based on a K-means clustering analysis, the studied population was clustered into three groups. People in cluster 1 showed a higher dependence on in-person grocery shopping for food, people in cluster 2 depended more on both in-person grocery shopping and in-house storage, and people in cluster 3 depended mostly on online food ordering and delivery. There were 1060 (53.2\%), 649 (32.5\%), and $285(14.3 \%)$ participants in clusters 1, 2, and 3, respectively. People in cluster 3 showed a significantly higher HDDS $(9.5 \pm 2.0)$ compared with people in clusters $1(9.2 \pm 1.9)$ and $2(9.1 \pm 2.0)$, $(p=0.021)$.

Regarding the dietary behaviors used to cope with COVID-19 in the post-lockdown time, there was a total of $661(33.1 \%)$ participants who used dietary supplements (vitamin C, probiotics, and other dietary supplements) and functional food (such as ginseng and Ganoderma) to cope with COVID-19, and a small proportion of the studied participants still used drinking alcohol and vinegar. Participants who chose to take vitamin C, probiotics, other dietary supplements, and functional food to cope with COVID-19 had a significant higher HDDS (Table 3). 
Table 3. Comparison of the HDDSs between participants with different coping behaviors against COVID-19 in the post-lockdown time.

\begin{tabular}{|c|c|c|c|c|}
\hline \multicolumn{2}{|c|}{ Behaviors to Cope with COVID-19 } & \multirow{3}{*}{$\begin{array}{c}N(\%) \\
503(25.2) \\
1491(74.8)\end{array}$} & \multirow{3}{*}{$\begin{array}{c}\text { HDDS (Mean } \pm \text { SD) } \\
9.5 \pm 1.9 \\
9.1 \pm 2.0\end{array}$} & \multirow{3}{*}{$\begin{array}{c}p \\
<0.001\end{array}$} \\
\hline Intake of vitamin $C$ & Yes & & & \\
\hline Intake of vitamin C & No & & & \\
\hline \multirow{2}{*}{ Intake of a probiotic } & Yes & 257 (12.9) & $9.6 \pm 2.0$ & \multirow{2}{*}{0.001} \\
\hline & No & $1737(87.1)$ & $9.1 \pm 1.9$ & \\
\hline \multirow{2}{*}{ Intake of other health products } & Yes & $166(8.3)$ & $9.7 \pm 1.9$ & \multirow{2}{*}{$<0.001$} \\
\hline & No & $1828(91.7)$ & $9.2 \pm 2.0$ & \\
\hline \multirow{2}{*}{ Intake of alcohol } & Yes & $105(5.3)$ & $9.5 \pm 2.3$ & \multirow{2}{*}{0.078} \\
\hline & No & $1889(94.7)$ & $9.2 \pm 1.9$ & \\
\hline \multirow{2}{*}{ Intake of vinegar } & Yes & $196(9.8)$ & $9.5 \pm 2.1$ & \multirow{2}{*}{0.057} \\
\hline & No & $1798(90.2)$ & $9.2 \pm 1.9$ & \\
\hline
\end{tabular}

Regarding the recommended dietary behaviors, in the post-lockdown time, $44.2 \%, 44.8 \%, 93.8 \%$, and $43.3 \%$ of participants who followed individual food serving, using serving chopsticks and spoons, cooking food fully, and preparing raw and cooked food separately recommendations, respectively. People who used serving chopsticks and spoons and prepared raw and cooked food separately had a significantly higher HDDS (Table 4).

Table 4. Comparison of the HDDSs of participants with different behaviors regarding food safety.

\begin{tabular}{ccccc}
\hline Behaviors Regarding food Safety & & $N(\%)$ & HDDS (Mean \pm SD) & $p$ \\
\hline \multirow{2}{*}{ Individual food servings } & Yes & $882(44.2)$ & $9.2 \pm 2.0$ & 0.386 \\
& No & $1112(55.8)$ & $9.2 \pm 1.9$ & \multirow{2}{*}{0.006} \\
\hline \multirow{2}{*}{ Using serving chopsticks and spoons } & Yes & $894(44.8)$ & $9.3 \pm 2.0$ & \multirow{2}{*}{0.084} \\
& No & $1100(55.2)$ & $9.1 \pm 1.9$ & \multirow{2}{*}{ Fully cooked food } \\
& Yes & $1930(96.8)$ & $9.2 \pm 1.9$ & $<0.001$ \\
\hline \multirow{2}{*}{ Preparing raw and cooked food separately } & No & $64(3.2)$ & $9.8 \pm 2.2$ & $8.8 \pm 2.0$ \\
\hline
\end{tabular}

\subsection{Multivariable Analysis of the Dietary Behaviors Associated with the HDDS}

As part of the multivariable analysis (Table 5), after adjusting for family income and living areas (whether the areas had a second outbreak), the increased online food purchasing, increased consumption of seafood, both increased and decreased consumption of imported frozen food, increased intake of raw food, and intake of dietary supplements (including vitamin C, probiotics, and other supplements and health products) were associated with a higher HDDS. People who did not follow the behaviors of using serving chopsticks and spoons or preparing raw and cooked food separately had a lower HDDS. 
Table 5. Multivariable analysis of dietary behaviors associated with the HDDS.

\begin{tabular}{|c|c|c|c|c|c|}
\hline \multirow{2}{*}{ Behaviors } & & \multicolumn{4}{|c|}{ HDDS } \\
\hline & & Crude $\beta(95 \% \mathrm{CI})$ & $p$ & Adjusted- $\beta(95 \% \mathrm{CI})^{\text {a }}$ & $p$ \\
\hline \multicolumn{6}{|c|}{ Dietary behavior changes compared with the same period in 2019} \\
\hline \multirow{3}{*}{ Consuming seafood ${ }^{a}$} & Increased & $0.45(0.15,0.76)$ & 0.004 & $0.39(0.09,0.70)$ & 0.010 \\
\hline & Stay the same & Reference & & Reference & \\
\hline & Decreased & $-0.05(-0.24,0.13)$ & 0.567 & $-0.00(-0.19,0.18)$ & 0.962 \\
\hline \multirow{3}{*}{ Consuming imported frozen food ${ }^{a}$} & Increased & $0.67(0.18,1.15)$ & 0.008 & $0.56(0.07,1.04)$ & 0.026 \\
\hline & Stay the same & Reference & & Reference & \\
\hline & Decreased & $0.26(0.08,0.44)$ & 0.004 & $0.27(0.09,0.44)$ & 0.003 \\
\hline \multirow{3}{*}{ Consuming raw food ${ }^{a}$} & Increased & $0.79(0.32,1.27)$ & 0.001 & $0.74(0.27,1.21)$ & 0.002 \\
\hline & Stay the same & Reference & & Reference & \\
\hline & Decreased & $0.11(-0.07,0.29)$ & 0.244 & $0.08(-0.10,0.26)$ & 0.359 \\
\hline \multicolumn{6}{|c|}{ Food-purchasing behaviors } \\
\hline \multirow{3}{*}{ Food purchasing methods ${ }^{b}$} & Cluster 1 & Reference & & Reference & \\
\hline & Cluster 2 & $-0.26(-0.22,0.16)$ & 0.786 & $-0.01(-0.20,0.18)$ & 0.940 \\
\hline & Cluster 3 & $0.40(0.14,066)$ & & $0.29(0.02,0.55)$ & 0.035 \\
\hline \multicolumn{6}{|c|}{ Dietary behaviors used to cope with COVID-19 in the post-lockdown time } \\
\hline \multirow{2}{*}{ Intake of health products ${ }^{c}$} & Yes & $0.39(0.21,0.57)$ & $<0.001$ & $0.31(0.19,0.42)$ & $<0.001$ \\
\hline & No & Reference & & Reference & \\
\hline \multicolumn{6}{|c|}{ Recommended dietary behaviors to prevent foodborne disease } \\
\hline \multirow{2}{*}{ Using serving chopsticks and spoons } & Yes & Reference & & Reference & \\
\hline & No & $-0.24(-0.41,-0.07)$ & 0.006 & $-0.29(-0.46,-0.11)$ & 0.001 \\
\hline \multirow{2}{*}{ Preparing raw and cooked food separately } & Yes & Reference & & Reference & \\
\hline & No & $-0.54(-0.73,-0.36)$ & $<0.001$ & $-0.52(-0.70,-0.34)$ & $<0.001$ \\
\hline
\end{tabular}

${ }^{a}$ Adjusted for family income and living in areas where a second outbreak episode occurred. ${ }^{b}$ People in cluster 1 showed a higher dependence on in-person grocery shopping for food, people in cluster 2 depended more on both in-person grocery shopping and in-house storage, and people in cluster 3 depended mostly on online food ordering and deliveries. ${ }^{\mathrm{c}}$ The health products included vitamin C, probiotics, and other dietary supplements.

\section{Discussion}

With the subsequent development of a global pandemic, COVID-19 has significantly changed the lifestyles and behaviors of people temporarily and may also bring permanent effects. Comparing data with that from the first stage of the survey that took place during the lockdown, the current second stage of the study showed that people still followed certain dietary behaviors to cope with COVID-19. Meanwhile, new dietary behaviors were acquired to help prevent infection. These behaviors were directly associated with the dietary diversity in Chinese adults.

\subsection{Dietary Behavior Changes Compared with 2019}

The WHO has specifically said that there is no evidence of food having been a source of infection during the pandemic, either through eating or touching contaminated food [19]. At the same time, however, some recent outbreaks of COVID-19 were reported to be closely related to food or markets $[16,20,21]$, and many countries, including China, had reported finding traces of the virus on packages of frozen food in the market [22,23]. Therefore, it is not difficult to see that, compared with the same period in 2019, this study showed more than half of participants reported that they decreased their eating at restaurants $(61.6 \%)$ and their consumption of seafood $(53.1 \%)$, as well as importing frozen food $(57.1 \%)$ and raw food $(60.5 \%)$, and at the same time, more people preferred to choose self-cooking at home $(64.8 \%)$. One newly released preprint on the biorxiv website shows that the coronavirus can survive on the surface of frozen food for up to three months, which indicates food-borne viruses are at least theoretically possible [24]. In this case, on the one hand, Chinese residents spontaneously reduced their intake of raw and frozen food, the frequency of eating outside, and the consumption of takeaways. On the other hand, many places removed frozen food from market storage, especially imported frozen seafood, which greatly impaired food accessibility [25]. It is also worth noting that an insufficient intake of seafood in the Chinese population had also been observed in a previous study conducted before the COVID-19 pandemic and during lockdown time $[8,26]$. The exacerbating decreased intake 
of seafood may bring potential nutrition concerns, especially for the ones who were living in inland areas, where most of their fish and seafood consumption depended on frozen food.

\subsection{Dietary Behavior Changes Compared with the Lockdown Period in the COVID-19 Pandemic}

When comparing the behaviors in the post-lockdown period with those during the lockdown, we found that the rate of using dietary supplements and functional food to cope with COVID-19 was not greatly changed in participants between stage $1(37.7 \%)$ and stage $2(33.1 \%)$ [8]. It is worth noting that irrational and unhealthy dietary behaviors still existed in a small proportion of participants. Although there was a great decline in people who chose to drink alcohol and vinegar to cope with COVID-19 compared with stage 1 [8], there were still $5.3 \%$ and $9.8 \%$ of participants, respectively, doing these behaviors in the post-lockdown period. The Chinese government had already dismissed the rumor that drinking alcohol or vinegar could prevent coronavirus as early as 22 January 2020 [27]. It seems that health education is still urgently needed and should be continued in any protracted COVID-19 pandemic.

\subsection{Dietary Behaviors Regarding Food Safety}

The dietary behaviors regarding food safety were also investigated. Cooking food fully and preparing raw and cooked food separately were always the key messages in food safety education to avoid food-borne disease [28,29]. In the current study, most participants followed the advice to cook food fully. However, $31.9 \%$ of participants did not prepare raw and cooked food separately, which may easily cause food contamination. In addition, according to the lessons learned from COVID-19, China currently encourages the public to use serving chopsticks and spoons and to adopt individual food servings [13]. However, since these behaviors are very different from traditional Chinese customs, this study found that more than half of the participants did not follow these new behaviors. To minimize the risk of COVID-19 infection, changes in dietary behavior regarding food safety should be incorporated in the current work on health promotion.

\subsection{Food Purchasing Behaviors}

With the development of e-commerce, China has become a front-runner in online retail due to it having the largest online population growth in the world; online ordering services have already been integrated into our daily life [30]. The success of China's fight against the pandemic cannot be separated from the cooperation of the whole nation and citizens' voluntary isolation at home. Online food services were strong guarantees of food accessibility during the lockdown. As the Global Times reported on the COVID-19 pandemic, online delivery services of fresh food increased 2.5-4-fold during the lockdown in China [31]. Contactless online food shopping by the public was also encouraged in many other countries [32]. In stage 2, $14.3 \%$ of participants mostly depended on online food shopping and deliveries. However, based on the currently limited data on online food services in the pandemic, several concerns were raised, such as, in some countries, online food services provided more highly energy-dense food, the coverage of online services could not reach rural areas, vulnerable groups were restricted in their access to digital technology, and there are potential food safety issues [33,34].

\subsection{Dietary Behaviors Associated with the Diet Diversity}

Dietary behaviors could greatly affect people's dietary intake, nutrition, and health. This study revealed that participants who increased their intake of seafood and raw food (including fruit and vegetables) had a significantly higher HDDS. Although some frozen food and seafood samples were found to be contaminated with the coronavirus, the infection could be avoided by appropriately handling and dealing with the food. According to the U.S. Center for Disease Control and Prevention advice, the risk of infection with COVID-19 could be minimized by following basic steps for food safety [35]. The current survey also observed not only that an increased intake of imported frozen food was associated with a higher HDDS but that a decreased intake was also related to a higher 
HDDS. We infer that this may be because the decrease in imported frozen food could be replaced by similar local food, and people who decided not to choose imported frozen food may usually pay more attention to their nutrition and health. In addition, this study also demonstrated that people who followed healthier dietary behaviors, including choosing to use dietary supplements and functional food, using serving chopsticks and spoons, and preparing raw and cooked food separately, had a higher HDDS. This could be explained by recognizing that this group of people was more concerned about their health and followed a healthier lifestyle, including eating a diversified diet.

It is worth noting that in stage 1 of our survey, we showed that the online ordering of food could help people to maintain dietary diversity during the lockdown [8]. In the current stage of the survey, we further identified that people who depended mainly on the online purchasing of food during the post-lockdown period also had a better HDDS. Even so, after combining the data from the first and second stages of the survey, there are reasons to believe that under strict supervision and management of food safety, the use of online food services could serve as a flexible way to help people purchase food contactlessly and ensure nutrition in the COVID-19 era.

\subsection{Limitations}

For convenience and in line with the first stage of the survey, the HDDS was used in this study. This is the index that can reflect food accessibility, and to some extent, be used to predict the risk of malnutrition; however, some validation studies mentioned that the HDDS does not provide a reliable reflection of the household-level access to food [36] and it cannot quantitatively assess the sufficient or insufficient intake of nutrients. Based on the online anonymous survey methodology, unfortunately, we could not follow up on all the participants in stage 1 ; there were $24.2 \%$ of participants enrolled in both stages 1 and 2 . The socio-demographic characteristics were not the same, where differences were found in age, education level, and geographic living areas. In addition, some vulnerable groups who were not familiar with digital technology could not be reached, and the dietary behaviors of these populations might be very different. Regarding dietary behavior, we did not measure the rate of using serving chopsticks and spoons, individual servings, etc., before and during the lockdown. Although these behaviors are obviously not traditional Chinese customs, we could not estimate exactly how many people picked up these new rules during the COVID-19 pandemic.

\section{Conclusions}

With a longitudinal study design, we observed the changes in dietary behavior between the lockdown and post-lockdown periods. People followed certain behaviors to prevent disease, such as decreasing outside eating; decreasing their consumption of raw food, seafood, and imported frozen food; increasing the frequency of cooking at home. Some unhealthy and irrational behaviors, including drinking alcohol or vinegar to prevent infection, were decreased but still existed. Meanwhile, following recommendations, more than half of the people used serving chopsticks/spoons and adopted individual servings. These behaviors had certain impacts on the dietary diversity. To ensure food diversity, the online food ordering and delivery services were very helpful in both the lockdown and post-lockdown periods.

Based on the results of the current study, education on how to scientifically and safely obtain, purchase, deal with, and eat food is still greatly needed and should be carried out throughout the whole COVID-19 era, even during the post-lockdown period.

Supplementary Materials: The following are available online at http://www.mdpi.com/2072-6643/12/11/3269/s1, Table S1: Socio-demographic characteristics of participants in stages 1 and 2 of the nutritional survey.

Author Contributions: Conceptualization, A.Z.; methodology, A.Z.; formal analysis, A.Z., J.Z.; and Z.R.; investigation, Y.K., S.H. and Y.M.; writing-original draft preparation, A.Z., J.Z. and Z.L. writing-review and editing, K.L. and Y.Z. All authors have read and agreed to the published version of the manuscript.

Funding: This research received no external funding. 
Conflicts of Interest: The authors declare no conflict of interest.

\section{References}

1. World Health Organization. Coronavirus Disease (COVID-19) Pandemic. Available online: https://www. who.int/emergencies/diseases/novel-coronavirus-2019 (accessed on 17 September 2020).

2. Nicola, M.; Alsafi, Z.; Sohrabi, C.; Kerwan, A.; Al-Jabir, A.; Iosifidis, C.; Agha, M.; Agha, R. The Socio-Economic Implications of the Coronavirus Pandemic (COVID-19): A review. Int. J. Surg. 2020, 78, 185-193. [CrossRef] [PubMed]

3. Global Times. Wuhan Lockdown Ends. Available online: https://www.globaltimes.cn/content/1184940.shtml (accessed on 15 September 2020).

4. Global Times. Beijing Reports New Domestic COVID-19 Infection after 55 Consecutive Days of Zero Cases. Available online: https://www.globaltimes.cn/content/1191313.shtml (accessed on 15 September 2020).

5. China Daily. Xinjiang Reports 5 New Local COVID-19 Cases. Available online: http://www.chinadaily.com. cn/a/202007/17/WS5f114702a31083481725a48e.html (accessed on 15 September 2020).

6. Yunnan Daily. Press Conference on COVID-19 Prevention and Control was Held in Ruili City, Yunnan Province. Available online: http://yndaily.yunnan.cn/html/2020-09/15/content_1368720.htm?div=-1 (accessed on 17 September 2020).

7. National Health Commission of the People Republic of China. Knowledge on Preventing COVID-19. Available online: http://www.nhc.gov.cn/xcs/kpzs/202005/bfb628ac14b54c4fa591951e452c3160. shtml (accessed on 17 September 2020).

8. Zhao, A.; Li, Z.; Ke, Y.; Huo, S.; Ma, Y.; Zhang, Y.; Zhang, J.; Ren, Z. Dietary Diversity among Chinese Residents during the COVID-19 Outbreak and Its Associated Factors. Nutrients 2020, 12, 1699. [CrossRef] [PubMed]

9. Sidor, A.; Rzymski, P. Dietary choices and habits during COVID-19 lockdown: Experience from Poland. Nutrients 2020, 12, 1657. [CrossRef] [PubMed]

10. Scarmozzino, F.; Visioli, F. Covid-19 and the subsequent lockdown modified dietary habits of almost half the population in an Italian sample. Foods 2020, 9, 675. [CrossRef] [PubMed]

11. Ruiz-Roso, M.B.; Padilha, P.D.C.; Mantilla-Escalante, D.C.; Ulloa, N.; Brun, P.; Acevedo-Correa, D.; Peres, W.A.F.; Martorell, M.; Aires, M.T.; Cardoso, L.D.O.; et al. Covid-19 Confinement and Changes of Adolescent's Dietary Trends in Italy, Spain, Chile, Colombia and Brazil. Nutrients 2020, 12, 1807. [CrossRef] [PubMed]

12. Khubchandani, J.; Kandiah, J.; Saiki, D. The COVID-19 Pandemic, Stress, and Eating Practices in the United States. Eur. J. Investig. Health Psychol. Educ. 2020, 10, 950-956. [CrossRef]

13. Zhang, J.; Zhang, Y.; Huo, S.; Ma, Y.; Ke, Y.; Wang, P.; Zhao, A. Emotional Eating in Pregnant Women during the COVID-19 Pandemic and Its Association with Dietary Intake and Gestational Weight Gain. Nutrients 2020, 12, 2250. [CrossRef] [PubMed]

14. Malekian, S. Iran Confronts Deadly Alcohol Crisis in Midst of Dealing with Coronavirus. Available online: https://abcnews.go.com/Health/iran-confronts-deadly-alcohol-crisis-midst-dealingcoronavirus/story?id=69842613 (accessed on 17 September 2020).

15. National Health Commission of the People Republic of China. Health Knowledge on Preventing COVID-19. Available online: http://www.nhc.gov.cn/xcs/kpzs/202005/4200755fd3f94c6a84613b18b846d912. shtml (accessed on 17 September 2020).

16. CCTV. COVID-19: Beijing's 36 New Cases All Linked to Xinfadi Market. Available online: http://english.cctv. com/2020/06/14/ARTIzFN9yNmKQsAVsLwHfXhp200614.shtml (accessed on 15 September 2020).

17. Sina News. Available online: http://k.sina.com.cn/article_1737737970_6793c6f201900xgqo.html?from=news\& subch=onews (accessed on 15 September 2020).

18. National Health Commission of People's Republic of China. Coronavirus Disease Situation Reports. Available online: http://www.nhc.gov.cn/xcs/yqtb/list_gzbd.shtml (accessed on 31 August 2020).

19. World Health Organization. Q\&A: Food Safety and Nutrition related to COVID-19. Available online: https://www.who.int/emergencies/diseases/novel-coronavirus-2019/question-and-answers-hub/q-a-detail/ food-safety-and-nutrition (accessed on 17 September 2020). 
20. Texas Observer. COVID-19 Cases Now Tied to Meat Plants in Rural Texas Counties Wracked with Coronavirus. Available online: https://www.texasobserver.org/east-texas-coronavirus-chicken/ (accessed on 15 September 2020).

21. Dyal, J.W.; Grant, M.P.; Broadwater, K.; Bjork, A.; Waltenburg, M.A.; Gibbins, J.D.; Hale, C.; Silver, M.; Fischer, M.; Steinberg, J.; et al. COVID-19 Among Workers in Meat and Poultry Processing Facilities-19 States, April 2020. MMWR. Morb. Mortal. Wkly. Rep. 2020, 69. [CrossRef] [PubMed]

22. SilkRoadPost. Nine Regions Found COVID-19 on Frozen Food in 40 days. Available online: https://huacheng. gz-cmc.com/pages/2020/08/14/33f55535537343aea7a5d07d48abb6c0.html (accessed on 15 September 2020).

23. The Standard. Covid Found on Brazil Frozen Chicken Wings. Available online: https: //www.thestandard.com.hk/section-news/section/11/221906/Covid-found-on-Brazil-frozen-chicken-wings (accessed on 15 September 2020).

24. Fishe, D.; Reilly, A.; Zheng, A.; Cook, A.; Anderson, D. Seeding of outbreaks of COVID-19 by contaminated fresh and frozen food. Environ. Chem. Lett. 2020. [CrossRef]

25. China Aquatic Products Processing and Marketing Alliance. The Global Seafood Trade Has Been Wreaked Havoc. Available online: http://www.cappma.org.cn/view.php?id=4756 (accessed on 17 September 2020).

26. Su, C.; Wang, Z.H.; Jia, X.F.; Zhang, B.; Ding, G.Q. Consumption of marine food consumption among adults aged 18-59 years old in 15 provinces of China in 2015. Acta Nutr. Sinca 2018, 1, 23-26.

27. Sohu News. Smoking, Alcohol Drinking Cannot Help for Preventing the Corona Virus. Available online: https://www.sohu.com/a/368530580_162522 (accessed on 20 April 2020).

28. World Health Organization. Five Keys to Safer Food Manual; WHO Press: Geneva, Switzerland, 2006.

29. Chinese Nutrition Society. Food and Nutrition Guidelines in COVID-19 Pandemic. Available online: https://www.cnsoc.org/othernews/532000207.html (accessed on 20 April 2020).

30. Maimaiti, M.; Zhao, X.; Jia, M.; Ru, Y.; Zhu, S. How we eat determines what we become: Opportunities and challenges brought by food delivery industry in a changing world in China. Eur. J. Clin. Nutr. 2018, 72, 1282-1286. [CrossRef] [PubMed]

31. Global Times. How China Guarantee the Food Supply in COVID-19 Pandemic. Available online: https: //oversea.huanqiu.com/article/3xuCwhiZ0ZJ (accessed on 19 September 2020).

32. Government of Canada. Coronavirus Disease (COVID-19) and Food Safety. Available online: https://www. canada.ca/en/health-canada/services/food-nutrition/food-safety/covid19.html\#safe_home_food (accessed on 19 September 2020).

33. Gao, X.; Shi, X.; Guo, H.; Liu, Y. To buy or not buy food online: The impact of the COVID-19 epidemic on the adoption of e-commerce in China. PLOS ONE 2020, 15, 0237900. [CrossRef]

34. Almandoz, J.P.; Xie, L.; Schellinger, J.N.; Mathew, M.S.; Gazda, C.; Ofori, A.; Kukreja, S.; Messiah, S.E. Impact of COVID-19 Stay-at-Home Orders on Weight-Related Behaviours among Patients with Obesity. Clin. Obes. 2020, 10, 12386. [CrossRef] [PubMed]

35. Centers of Disease Control and Prevention. Food and Coronavirus Disease 2019 (COVID-19). Available online: https://www.cdc.gov/coronavirus/2019-ncov/daily-life-coping/food-and-COVID-19.html\#risk (accessed on 17 September 2020).

36. Vellema, W.; Desiere, S.; Dhaese, M. Verifying Validity of the Household Dietary Diversity Score: An Application of Rasch Modeling. Food Nutr. Bull. 2016, 37, 27-41. [CrossRef] [PubMed]

Publisher's Note: MDPI stays neutral with regard to jurisdictional claims in published maps and institutional affiliations.

(C) 2020 by the authors. Licensee MDPI, Basel, Switzerland. This article is an open access article distributed under the terms and conditions of the Creative Commons Attribution (CC BY) license (http://creativecommons.org/licenses/by/4.0/). 